\title{
Identification of genetic factors underlying persistent pulmonary hypertension of newborns in a cohort of Chinese neonates
}

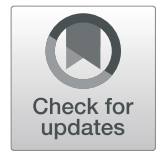

Xu Liu ${ }^{1,2+}$, Mei Mei ${ }^{3 \dagger}$, Xiang Chen ${ }^{2}$, Yulan $\mathrm{Lu}^{4}$, Xinran Dong ${ }^{4}$, Liyuan $\mathrm{Hu}^{2}$, Xiaojing $\mathrm{Hu}^{2}$, Guoqiang Cheng ${ }^{2}$, Yun $\mathrm{Cao}^{2}$, Lin Yang ${ }^{1,4^{*}}$ and Wenhao Zhou ${ }^{1,2,4^{*}}$

\begin{abstract}
Background: Persistent pulmonary hypertension of the newborn (PPHN) is a severe clinical problem among neonatal intensive care unit (NICU) patients. The genetic pathogenesis of PPHN is unclear. Only a few genetic polymorphisms have been identified in infants with PPHN. Our study aimed to investigate the potential genetic etiology of PPHN.

Methods: This study recruited PPHN patients admitted to the NICU of the Children's Hospital of Fudan University from Jan 2016 to Dec 2017. Exome sequencing was performed for all patients. Variants in reported PPHN/pulmonary arterial hypertension (PAH)-related genes were assessed. Single nucleotide polymorphism (SNP) association and gene-level analyses were carried out in 74 PPHN cases and 115 non-PPHN controls with matched baseline characteristics.

Results: Among the patient cohort, 74 (64.3\%) patients were late preterm and term infants ( $\geq 34$ weeks gestation) and 41 (35.7\%) were preterm infants ( $<34$ weeks gestation). Preterm infants with PPHN exhibited low birth weight and a high frequency of bronchopulmonary dysplasia, respiratory distress syndrome (RDS) and mortality. Nine patients (only one preterm infant) were identified as harboring genetic variants, including three with pathogenic/likely pathogenic variants in TBX4 and BMPR2 and six with variants of unknown significance in BMPR2, SMAD9, TGFB1, KCNA5 and TRPC6. Three SNPs (rs192759073, rs1047883 and rs2229589) in CPS1 and one SNP (rs1044008) in NOTCH3 were significantly associated with PPHN $(p<0.05)$. CPS1 and SMAD9 were identified as risk genes for PPHN $(p<0.05)$.

Conclusions: In this study, we identified genetic variants in PPHN patients, and we reported CPS1, NOTCH3 and SMAD9 as risk genes for late preterm and term PPHN in a single-center Chinese cohort. Our findings provide additional genetic evidence of the pathogenesis of PPHN and new insight into potential strategies for disease treatment.
\end{abstract}

Keywords: Persistent pulmonary hypertension of the newborn, Exome sequencing, Chinese, PAH genes, Genetic polymorphisms, Early diagnosis

\footnotetext{
* Correspondence: linyang09@fudan.edu.cn; zhouwenhao@fudan.edu.cn

${ }^{+}$Xu Liu and Mei Mei contributed equally to this work.

${ }^{1}$ Clinical Genetic Center, Children's Hospital of Fudan University, 399

Wanyuan Road, Shanghai 201102, China

Full list of author information is available at the end of the article
}

(c) The Author(s). 2019 Open Access This article is distributed under the terms of the Creative Commons Attribution 4.0 International License (http://creativecommons.org/licenses/by/4.0/), which permits unrestricted use, distribution, and reproduction in any medium, provided you give appropriate credit to the original author(s) and the source, provide a link to the Creative Commons license, and indicate if changes were made. The Creative Commons Public Domain Dedication waiver (http://creativecommons.org/publicdomain/zero/1.0/) applies to the data made available in this article, unless otherwise stated. 


\section{Background}

Persistent pulmonary hypertension of the newborn (PPHN) is caused by a failure in the normal circulatory transition at birth and is characterized by elevated pulmonary vascular resistance (PVR), which leads to right-to-left shunting and hypoxemia. The incidence of PPHN ranges from 2 to 6 per 1000 live births and the mortality rate is $10-20 \%$ [1]. PPHN can be idiopathic or may be caused by multiple pulmonary diseases including perinatal asphyxia, meconium aspiration syndrome (MAS), respiratory distress syndrome (RDS), pulmonary dysplasia and congenital diaphragmatic hernia [2].

To date, only a few genetic polymorphisms have been identified in infants with PPHN. Pearson et al. first found that a T1405 N variant of carbamoyl phosphate synthetase I (CPS1) exhibited a different distribution between infants with PPHN and the general population [3]. A homozygous missense variant (L326R) in the $A B C A 3$ gene was identified in a newborn with severe hypoxemic respiratory failure and refractory pulmonary hypertension [4]. Single nucleotide polymorphisms (SNPs) in the corticotropin-releasing hormone receptor 1 (CRHR1) and corticotropinreleasing hormone-binding protein $(C R H B P)$ genes were significantly associated with PPHN [5]. Most recently, rs2070699 in endothelin 1 (EDN1) was found to increase the risk of PPHN with respiratory distress [6].

PPHN is a subgroup of pulmonary arterial hypertension (PAH), which is a complex disorder characterized by elevation of PVR and failure of right heart function. It is a common complication of many clinical diseases. PAH is classified into five types according to the pathogenesis of the disease (WHO classification) and PPHN is a specific group [7]. Pathogenic variants among several genes have been reported in PAH patients exhibiting both adulthood onset and childhood onset. Bone morphogenic protein receptor type 2 (BMPR2), a member of the transforming growth factor beta (TGF- $\beta$ ) superfamily, is associated with $70 \%$ of familial pulmonary arterial hypertension (FPAH)/heritable pulmonary arterial hypertension (HPAH) cases and $20 \%$ of idiopathic pulmonary hypertension (IPAH) cases [8]. SMAD9 [9], CAV1 [10], KCNK3 [11] are also known PAH genes listed in the Online Mendelian Inheritance in Man (OMIM) database. Hereditary hemorrhagic telangiectasia (HHT) gene variants in the activin receptor-like kinase 1 (ACVRL1) [12] and endoglin (ENG) [13] have been identified in PAH patients. To date, no causal genes for PPHN have been reported, and the genetic etiology remains unclear. We suggest that the genetic pathogenesis of PPHN may share some similarities with $\mathrm{PAH}$ in adults and children.

Therefore, in the present study, we applied clinical exome sequencing to investigate the genetic etiology of PPHN in 115 Chinese patients. We aimed to identify causal variants in reported PPHN/PAH-related genes and genetic risk polymorphisms for PPHN patients.

\section{Methods \\ Study participants}

In this study, neonates who were admitted to the neonatal intensive care unit (NICU) of the Children's Hospital of Fudan University from January 2016 to December 2017 were recruited. The inclusion criteria were neonates with hypoxemic respiratory failure with a clinical diagnosis of pulmonary hypertension within 28 days after birth. Neonates with PPHN were diagnosed based on clinical and echocardiographic data. The criteria used for diagnosis was from previously published work by Alano et al. [14]: 1) the clinical criteria included a preductal/postductal oxygen saturation difference of $>10 \%$, and 2) the echocardiographic criteria included a structurally normal heart and elevated pulmonary artery pressure (PAP). The last criterion was considered to be present if there was either right-to-left or bidirectional flow across the patent ductus arteriosus or foramen ovale, or systolic pulmonary arterial pressure was greater than or equal to the systemic blood pressure according to Doppler measurement of the tricuspid-regurgitation jet. Neonates with congenital anomalies or structural congenital heart disease other than patent ductus arteriosus and patent foramen ovale were excluded.

Infants who did not have pulmonary hypertension were collected as non-PPHN controls. Late preterm and term PPHN cases and control infants with matched baseline characteristics were included for further casecontrol analysis. All guardians of the subjects included in this study were provided with appropriate informed consent. This study was approved by the ethics committee of the Children's Hospital of Fudan University.

\section{Clinical exome sequencing}

Genomic DNA was extracted from whole blood using the QIAamp DNA Blood Mini Kit (Qiagen, Hilden, Germany). The DNA concentration was measured using a Nano-Drop spectrophotometer (ND-1000, Thermo Fisher Scientific Inc., Waltham, MA, USA). The clinical exome panel used for sequencing, which covered 2742 genes causing inherited diseases, was generated using the Agilent ClearSeq Inherited Disease Kit (Agilent Technologies, Santa Clara, CA) and Illumina Cluster and SBS Kits (Illumina Inc., San Diego, CA, USA). Sequencing was performed on the Illumina HiSeq 2000/ 2500 platform (Illumina Inc., San Diego, CA, USA). Clean reads were aligned to the reference human genome (UCSC hg19) by the Burrows-Wheeler Aligner (BWA; v.0.5.9-r16). After quality control, variants were obtained using GATK. 


\section{Variant annotation and classification}

Variants were annotated by using ANNOVAR [15] and VEP [16] software, and the Human Gene Mutation Database (HGMD, professional version) and ClinVar database (https://www.ncbi.nlm.nih.gov/clinvar/). Missense variants were evaluated with SIFT [17], PolyPhen2 [18] and MutationTaster [19]. Common variants with a minor allele frequency (MAF) $>0.01$ were excluded based on the Exome Aggregation Consortium (ExAC) database (http://exac.broadinstitute.org/), 1000 Genomes database (http://www.internationalgenome.org/) and our in-house database. Synonymous and intronic variants falling outside the $+/-15$ bp boundaries of exons were also discarded. MAFs for all variants were collected from the ExAC database and the Genome Aggregation Database (GnomAD, http://gnomad.broadinstitute.org/). Causal variants were further selected in 25 reported PPHN/PAH disease-related genes (Additional file 1: Table S1). Only variants with a very low frequency $(\mathrm{MAF}<0.005)$ in both the overall and East Asian populations and were absent from infants without PPHN in our in-house database (including 24,336 samples) were further considered to be pathogenic. The pathogenicity of the variants was defined based on the American College of Medical Genetics and Genomics (ACMG) criteria [20]. Specifically, a case was classified as molecularly diagnosed when the identified pathogenic or likely pathogenic $(\mathrm{P} / \mathrm{LP})$ variants were truncating variants or reported missense variants detected in a disease gene that sufficiently explained the phenotypes of the studied individual. Variants with unknown significance (VUSs) were associated with one or more clinical phenotypes of patients and were absent or present in the GnomAD/ ExAC databases with low frequencies.

\section{SNP calling and quality control}

Only SNPs with high confidence (depth $\geq 10$, call ratio $\geq$ 0.2 for heterogeneous variants and $\geq 0.6$ for homogeneous variants) that were present in at least one individual were selected for further analysis. The following filtering criteria were used to filter SNPs for case-control analysis: 1) missing rate $<0.2,2)$ MAF (PLINK) $>0.001$; 3) control Hardy-Weinberg equilibrium (HWE) (PLINK) > 0.001. SNPs within the sequencing coding region of 25 PPHN/PAH-related genes were included for further analysis. The workflow is shown in Fig. 1.

\section{Statistical analysis}

Differences in clinical characteristics in different study groups were analyzed using the t-test for continuous variables and the chi-square test for categorical variables. A two-sided type I error of 0.05 was used to test for statistical significance. The statistical analyses were performed with SPSS version 16 (SPSS Inc., Chicago, IL,

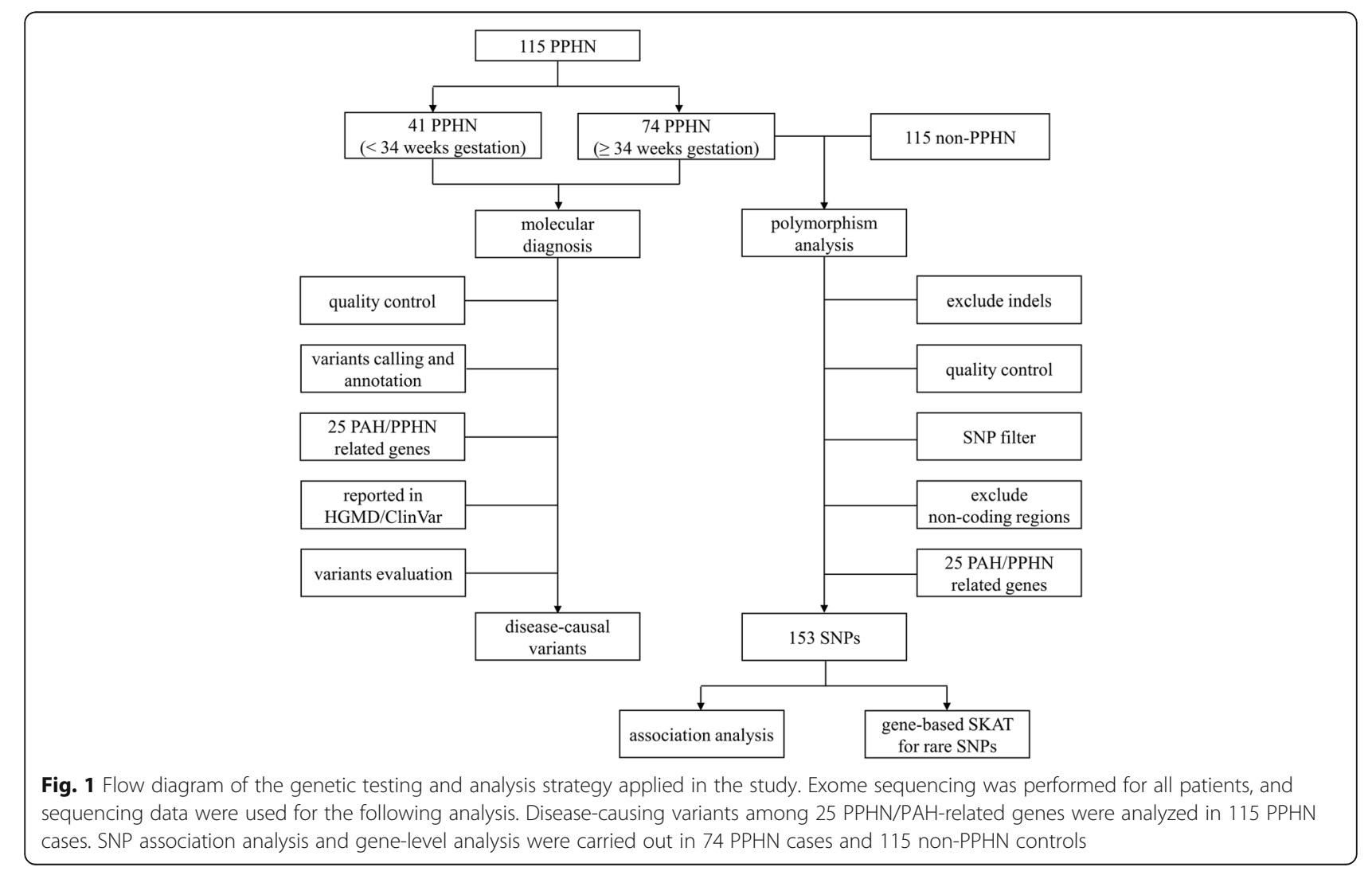


USA). The results of SNP association analysis using exome sequence data were analyzed with the chi-square test using PLINK software (http://zzz.bwh.harvard.edu/ plink/, version 1.07). Gene-level analysis using the Sequence Kernel Association test (SKAT) [21] in the $R$ package with default settings was carried out for rare SNPs $($ MAF < 0.05).

\section{Results}

\section{Patient characteristics}

A total of 115 infants diagnosed with PPHN were enrolled in this study. The average gestational age was 34.9 weeks, and the average birth weight was $2516.2 \mathrm{~g}$. The majority of the patients were male $(64,55.7 \%)$. The age at diagnosis for all patients ranged from 1 day to 5 days after birth, and the majority (110 patients) were diagnosed within 3 days. RDS (53, 46.1\%) and pneumonia $(44,38.3 \%)$ were two major primary diagnoses in PPHN cases. Among all cases, 17 were treated with inhaled nitric oxide (iNO), and 4 were treated with extracorporeal membrane oxygenation (ECMO). The patients' demographic and clinical characteristics are shown in Table 1 and Fig. 2a.

PPHN occurred in most $(64.3 \%, 74 / 115)$ of the late preterm and term infants ( $\geq 34$ weeks gestation), and $35.7 \%$ $(41 / 115)$ of the patients were preterm infants $(<34$ weeks gestation). Preterm infants exhibited lower birth weight, a higher incidence of RDS and were more likely to require longer ventilation treatment. iNO treatment was usually carried out in late preterm and term infants, and ECMO was only performed in late preterm and term infants. Specifically, 17 infants (16 infants $>34$ weeks gestation and 1 infant $<34$ weeks gestation) were treated with iNO, and 4 infants (all $>34$ weeks gestation) were treated with ECMO. Mortality was higher in preterm patients than late preterm and term infants ( $17.1 \%$ vs $9.5 \%)$.

\section{Sequencing results}

An average of 27.5 million effective reads were generated with an average sequencing depth of 230.98-fold per target in 115 PPHN patients and 115 non-PPHN controls. In total, $99.8 \%$ of the target region was covered, among which $99.6 \%$ was covered at least 10 -fold, and $99.2 \%$ was covered at least 20 -fold.

\section{Genetic variant identification PPHN/PAH-related genes}

In total, 9 phenotype related variants spanning 6 PAH-related genes were identified in 9 patients (7.8\%). The variants included $3 \mathrm{P} / \mathrm{LP}$ variants and $6 \mathrm{VUSs}$, among which two were reported disease-causing variants, and seven were novel. The $3 \mathrm{P} / \mathrm{LP}$ variants, including one stop-gain variant (c.1633G > T. p.G545X) in TBX4 and two reported PAH-

Table 1 Demographic and clinical characteristics of study population

\begin{tabular}{|c|c|c|c|c|c|c|}
\hline Characteristics & $\begin{array}{l}\text { All PPHN } \\
(n=115)\end{array}$ & $\begin{array}{l}\text { Preterm } \\
(<34 \text { weeks }) \\
\text { PPHN } \\
(n=41)\end{array}$ & $\begin{array}{l}\text { Late preterm } \\
\text { and term } \\
(\geq 34 \text { weeks) } \\
\text { PPHN } \\
(n=74)\end{array}$ & $\begin{array}{l}P \text { value } \\
(<34 \text { weeks } \\
\text { PPHN vs } \geq \\
34 \text { weeks PPHN) }\end{array}$ & $\begin{array}{l}\text { Non-PPHN } \\
(n=115)\end{array}$ & $\begin{array}{l}P \text { value } \\
\text { ( } \geq 34 \text { weeks } \\
\text { PPHN vs } \\
\text { non-PPHN) }\end{array}$ \\
\hline \multicolumn{7}{|l|}{ Baseline characteristics } \\
\hline Male gender, n(\%) & $64(55.7)$ & $24(58.5)$ & $40(54.1)$ & 0.6430 & $62(53.9)$ & 0.9849 \\
\hline Birth weight $(\mathrm{g})$, mean $( \pm \mathrm{SD})$ & $2516.2 \pm 1006.2$ & $1358.8 \pm 391.7$ & $3157.4 \pm 572.6$ & $<0.001$ & $3169.3 \pm 576.6$ & 0.8896 \\
\hline Gestational age (weeks), mean $( \pm S D)$ & $34.9 \pm 4.4$ & $29.7 \pm 2.3$ & $37.8 \pm 1.9$ & $<0.001$ & $38.2 \pm 1.4$ & 0.1649 \\
\hline Premature (<37 weeks), n(\%) & $60(52.2)$ & $41(100)$ & $19(25.7)$ & $<0.001$ & $17(14.8)$ & 0.0627 \\
\hline Caesarean, n(\%) & $76(66.1)$ & $24(58.5)$ & $52(70.3)$ & 0.2030 & $70(60.9)$ & 0.1873 \\
\hline \multicolumn{7}{|l|}{ Primary diagnosis for PPHN cases } \\
\hline Respiratory distress syndrome, n(\%) & $53(46.1)$ & $27(65.9)$ & $26(35.1)$ & 0.0015 & / & / \\
\hline Meconium aspiration syndrome, $\mathrm{n}(\%)$ & $10(8.7)$ & $0(0)$ & $10(13.5)$ & 0.0135 & / & / \\
\hline Pneumothorax, n(\%) & $17(14.8)$ & $3(7.3)$ & $14(18.9)$ & 0.0932 & / & / \\
\hline Pneumonia, n(\%) & $44(38.3)$ & $14(34.1)$ & $30(40.5)$ & 0.4992 & / & / \\
\hline Bronchopulmonary dysplasia, n(\%) & $10(8.7)$ & $9(22.0)$ & $1(1.4)$ & 0.0004 & / & / \\
\hline \multicolumn{7}{|l|}{ Treatment and prognosis for PPHN cases } \\
\hline Ventilation time (days), mean $( \pm S D)$ & $12.7 \pm 18.9$ & $22.2 \pm 26.9$ & $6.8 \pm 6.7$ & 0.0015 & / & / \\
\hline iNO, n(\%) & $17(14.8)$ & $1(2.4)$ & $16(21.6)$ & 0.0055 & / & / \\
\hline Vasoactive agents therapy, $\mathrm{n}(\%)$ & $64(55.7)$ & $19(46.3)$ & $45(60.8)$ & 0.1347 & / & / \\
\hline ECMO, n(\%) & $4(3.5)$ & $0(0)$ & $4(5.4)$ & 0.2952 & / & / \\
\hline Mortality, n(\%) & $14(12.2)$ & $7(17.1)$ & $7(9.5)$ & 0.2474 & / & / \\
\hline
\end{tabular}




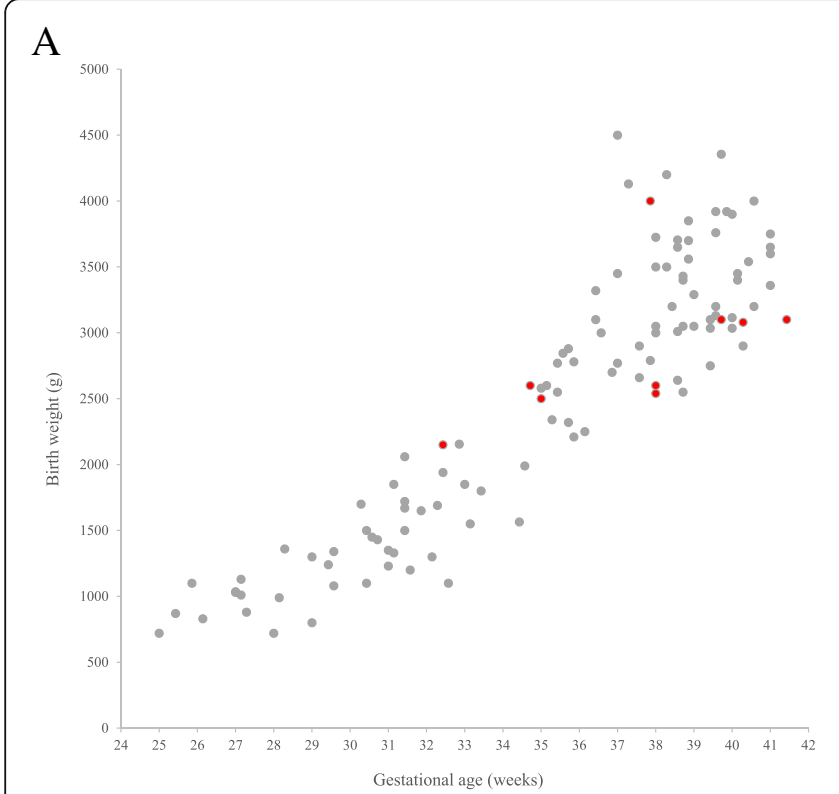

B

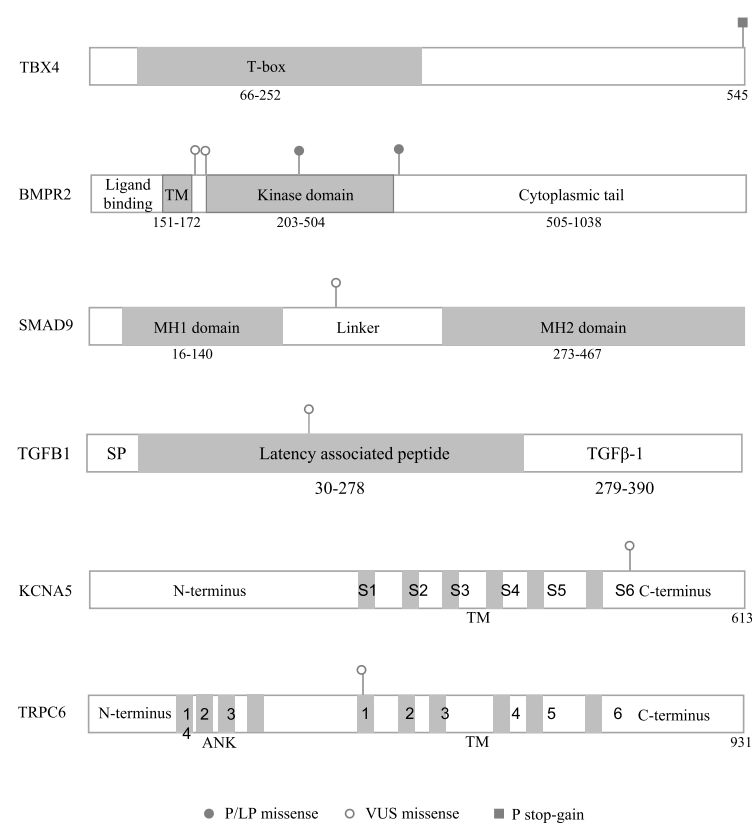

Fig. 2 Gestational age and birth weight of 115 PPHN patients and schematic representation of functional domains and locations of variants in the 6 PAH disease gene proteins. a Birth weight $(\mathrm{g})$ was plotted on the $y$ axis and gestational age was plotted on the $x$ axis. One dot represents one patient. Red dots were patients identified with genetic variants. b The figure shows variants in the TBX4, BMPR2, SMAD9, TGFB1, KCNA5 and TRPC6 proteins. Missense pathogenic/likely pathogenic (P/LP) variants are indicated with solid circles; missense variants with unknown significance (VUSs) are indicated with hollow circles; and one pathogenic stop-gain variant is indicated with a square. TM: transmembrane region; SP: signal peptide; ANK: ankyrin

associated missense variants (c.1535A > C, p.K512 T [22]; and c.1094G > A, p.R365H) [23] in BMPR2, were identified in three patients. K512 $\mathrm{T}$ was reported in one adult patient of western European origin with an age at onset of 19-50 years, and $\mathrm{R} 365 \mathrm{H}$ was reported in one adult IPAH patient in Spain. Two novel missense variants (c.565A $>$ G, p.M189 V; and c.596A > T, p.D199V) in BMPR2 and one novel missense variant (c.587C $>$ T, p.A196V) in SMAD9 were classified as VUSs. In addition, three VUSs in PAH genes (TGFB1 (c.426A > C, p.E142D), KCNA5 (c.1645G > T, p.D549Y) and TRPC6 (c.1327 T > A, p.F443I)) were also found individually in three patients. Detailed information on all variants is shown in Table 2 and Fig. 2 b.

\section{PPHN/PAH-unrelated genes}

In three patients who presented with other clinical features, $\mathrm{P} / \mathrm{LP}$ variants were identified in PPHN/PAH-unrelated genes. Two patients exhibited metabolic diseases: a boy with hypoglycemia carried a novel stop-gain variant (c.2331G > A, p.W777X) in $A B C C 8$ that was associated with hypoglycemia in infancy [MIM:240800], and a girl with metabolic acidosis carried two LP variants, including one stop-gain variant (c.3853C > T, p.Q1285X) and one splicing variant $(c .3303+1 G>C)$ in $O P L A H$, that were associated with 5-oxoprolinase deficiency [MIM:260005]. The other patient was a female with multiple arrhythmias, in whom a reported stop-gain variant $(\mathrm{c} .1096 \mathrm{C}>\mathrm{T}$,
p.R366X) in $K C N H 2$ related to long QT syndrome 2 [MIM:613688] and short QT syndrome 1 [MIM:609620] was identified.

\section{Clinical phenotypes in nine patients with PPHN/PAH- related genes}

Among the reported PPHN/PAH-related genes, TBX4 and BMPR2 were two major genetic factors in PPHN. Patients P001-P003 carried P/LP variants in these two genes. P001 was a girl with a stop-gain variant in TBX4 who presented with severe PPHN with a maximal oxygenation index (MOI) value of 27. She was treated with ventilation for 8 days and vasoactive agent therapy for 5 days. Patient P002 displayed a moderate phenotype with a PAP of $50 \mathrm{mmHg}$ and an MOI value of 12.5. She was treated with ventilation for 8 days, sildenafil therapy for 5 days and vasoactive agent therapy for 9 days. This girl was identified as harboring a pathogenic missense variant (K512 T) in BMPR2, located at the cytoplasmic tail of the protein. Patient P003 was a girl who exhibited a severe phenotype with a PAP of $82 \mathrm{mmHg}$ and an MOI value of 22.9. She was treated with ventilation for 21 days, sildenafil therapy for 11 days and vasoactive agent therapy for 15 days. The patient had been administered ECMO treatment for 15 days. An $\mathrm{R} 365 \mathrm{H}$ variant was identified in this patient. The residue is located in the kinase domain, which is important for kinase activity 


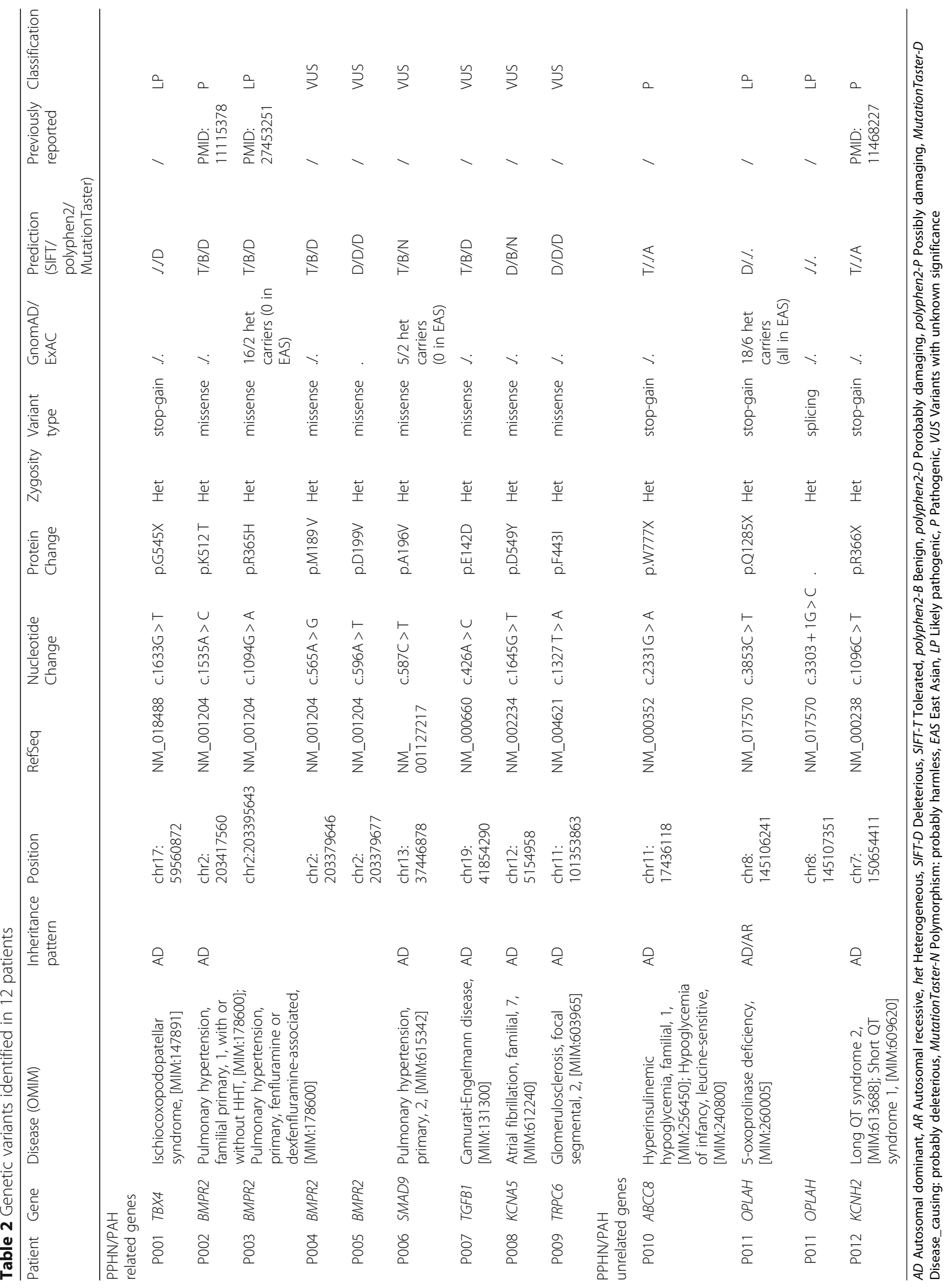


(Fig. 2b). This variant has been reported as a possibly pathogenic variant for PAH. Although this variant appears in GnomAD (16 heterogeneous carriers) and ExAC (2 heterogeneous carriers), it is absent in the East Asian population in these two databases and in our large internal database with 24,336 non-PPHN samples. Therefore, this variant was considered an LP for PPHN and needs to be further confirmed in the patients' family.

Patients P004 and P005, who exhibited the novel VUSs M189 V and D199V in BMPR2, located between the transmembrane region and the kinase domain of the protein, were diagnosed with mild PPHN. Patients P006 and P008P009, who harbored VUSs in SMAD9, KCNA5 and TRPC6, also displayed moderate to mild phenotypes. A boy P007 who carried an E142D variant in TGFB1 exhibited severe PPHN with an MOI value of 23.1. He was treated with ventilation for 11 days and vasoactive agent therapy for 7 days. This residue is located in the $\alpha 3$-helix of the ARM domain of the latency-associated peptide (LAP) region (Fig. 2b). LAP is required for homodimer assembly and protein secretion, and regulates the bioactivity of TGF- $\beta$ [24]. Detailed information on the clinical phenotypes is shown in Additional file 2: Table S2.

\section{Risk polymorphism identification}

We included 74 PPHN cases and 115 non-PPHN controls with matched clinical characteristics $(\geq 34$ weeks gestational age) in case-control analysis to exclude the influence of nongenetic risk factors. All baseline characteristics of the case and control groups were similar (Table 1). After quality control and SNP filtering, 153 SNPs remained in 25 PPHN/PAH-related genes. Three SNPs in CPS1 and one SNP in NOTCH3 were significantly associated with PPHN (Table 3). The most significant SNPs were rs192759073 in CPS1 and rs1044008 in NOTCH3 $(p=0.03)$. The other two SNPs in CPS1, rs1047883 and rs2229589 were in linkage disequilibrium $\left(\mathrm{LD}, r^{2}=1\right)$ and exhibited a relatively high frequency in public databases (0.45). We considered the first two SNPs (rs192759073 in CPS1 and rs1044008 in NOTCH3) to be better risk markers for PPHN. Gene-level analysis was performed for 128 rare SNPs $(\mathrm{MAF}<0.05)$ spanning 15 genes. Among these gene sets, CPS1 was associated with PPHN at $p=0.006$, which was consistent with the association of CPS1 SNPs and PPHN (Additional file 3: Table S3). SMAD9 was also associated with PPHN at $p=0.039$.

\section{Discussion}

PPHN is a severe clinical problem and accounts for $\sim 6 \%$ of our NICU patients. The role of genetics in the pathogenesis of PPHN remains elusive. The present study investigated the genetic contributions to the pathogenesis of PPHN in 115 Chinese PPHN patients using exome sequencing. Among all cases, 41 were preterm infants and 74 were late preterm and term infants. We identified three patients with P/LP variants in TBX4 and BMPR2 and six patients with VUSs in BMPR2 and 4 other reported PAHrelated genes. CPS1, NOTCH3 and SMAD9 were identified as important risk genes for late preterm and term PPHN through case-control analysis.

PPHN has generally been recognized to occur among late preterm and term infants, but studies have reported an increasing rate of PPHN in preterm infants [25]. In this study, most of the infants with PPHN were late preterm and term infants ( $\geq 34$ weeks gestation), and preterm infants also accounted for $35.7 \%$ of the patients. Among the 9 patients with genetic findings, only 1 patient with c.596A $>\mathrm{T}$ (p.D199V) in BMPR2 was born before 34 weeks gestation $(32+3$ weeks). The genetic diagnosis rates were different in the two groups $(8 / 74$ in the $\geq 34$-week gestation group vs $1 / 41$ in the $<34$-week gestation group). Our findings indicated that preterm complications play major roles in preterm infants with PPHN, while genetic factors have a greater effect on late preterm and term infants.

In terms of the genetic background of PPHN, previous studies have not found the disease-causing gene for PPHN patients thus far, and only polymorphisms in 5 genes have been reported to be associated with PPHN. PAH has been widely studied in both adults and children, and 20 genes have been associated with the development of the disease (Additional file 1: Table S1). The genetic etiology of PPHN in newborns is complex and unclear and may share some similarities with $\mathrm{PAH}$ in adults and children. In this study, we identified several variants in PAH-related genes, which verified that PAH

Table 3 Significant SNPs in PAH related genes with PPHN

\begin{tabular}{llllllllc}
\hline SNP & Position & Gene & Alleles & $\begin{array}{l}\text { MAF in } \\
\text { cases }\end{array}$ & $\begin{array}{l}\text { MAF in } \\
\text { controls }\end{array}$ & P value & $\begin{array}{l}\text { Frequency in } \\
\text { GnomAD_EAS }\end{array}$ & $\begin{array}{l}\text { Frequency } \\
\text { in ExAC_EAS }\end{array}$ \\
\hline rs192759073 & chr2:211438090 & CPS1 & C > T & 0.02027 & 0 & 0.030 & 0.007321 & 0.006702 \\
rs1044008 & chr19:15272001 & NOTCH3 & C > T & 0.02027 & 0 & 0.030 & 0.001627 & 0 \\
rs1047883 & chr2:211456637 & CPS1 & G > A & 0.5338 & 0.4261 & 0.041 & 0.4515 & 0.4534 \\
rs2229589 & chr2:211456639 & CPS1 & T > C & 0.5338 & 0.4261 & 0.041 & 0.4515 & 0.4534 \\
\hline
\end{tabular}

SNP rs 1047883 is in LD with rs2229589 $\left(r^{2}=1\right)$. MAF Minor allele frequency, EAS East Asian 
and PPHN potentially exhibit a common genetic pathogenesis. We also found three disease-causing genes in the three other patients. However, these genes were not associated with the development of pulmonary hypertension. Further studies are needed to investigate other potential disease-causing genes related to PPHN.

Among the genes identified in this study, several genetic variants in the BMP/TGF- $\beta /$ SMAD pathways were identified, including three $\mathrm{P} / \mathrm{LP}$ variants in $T B X 4$ and $B M P R 2$ and one VUS in TGFB1 related to severe clinical phenotypes in four patients. BMP/TGF- $\beta /$ SMAD signaling (especially $B M P R 2$ ) has been reported to be involved in the regulation of the proliferation and apoptosis of pulmonary arterial smooth muscle cells (PASMCs) and pulmonary arterial endothelial cells (PAECs) [26]. In a previous study of $\mathrm{PAH}, B M P R 2$ variants were more commonly found in females than males (3.6:1 ratio in adult-onset PAH cases and 1.7:1 ratio in pediatric-onset PAH cases) [27]. The sex ratio was similar (3:1, female: male ratio) in our study among the $4 B M P R 2$ variantcarrying PPHN patients. TBX4 is a member of the T-box genes that is important for the development of airway branching and the regulation of lung fibrosis. TBX4 variants have been reported in childhood-onset PAH patients [28] and might contribute to $\mathrm{PAH}$ by decreasing the activation of the BMP/TGF- $\beta / \mathrm{SMAD}$ signaling pathways [29]. TGFB1 (transforming growth factor $\beta 1$ ) is a member of the TGF- $\beta$ superfamily, whose members are important modulators of cell growth, inflammation and apoptosis. TGFB1 can suppress the proliferation and migration of endothelial and smooth muscle cells and thereby inhibit vascular remodeling. Variants in TGFB1 might affect its function and lead to pulmonary hypertension [30]. Both the TGF- $\beta$ and BMP signaling pathways ultimately converge on SMADs. One rare SMAD9 variant, A196V, located in the linker domain of the protein was identified in one patient (Fig. 2b). The linker region of SMAD9 is rendered shorter than those of other SMADs, which suppresses its transcriptional activity and ability to activate BMP signaling, while facilitating interaction with other molecules [31]. In addition, two ion channel genes, which might also play important roles in PAH, were identified in our patients. The Kv1.5 channel gene (KCNA5) is a pore-forming $\alpha$-subunit that forms a voltage-gated $\mathrm{K}^{+}$channel in PASMCs. Downregulation of KCNA5 causes membrane depolarization and increases the cytosolic $\mathrm{Ca}^{2+}$ concentration, resulting in pulmonary vasoconstriction, and pulmonary vascular remodeling [32]. A novel D549Y variant in KCNA5 was identified in one girl. The residue is located in the Clinker region following transmembrane domain segment 6. Another novel variant, F443I in TRPC6, was identified in another patient. TRPC6 is an important member of the TRPC channels of the transient receptor potential
(TRP) superfamily expressed in the lungs and PASMCs [33]. A SNP in the promoter region of TRPC6 has been demonstrated to increase the risk of IPAH by recruiting NF-kB [34].

Furthermore, we performed SNP association and genelevel analyses in $25 \mathrm{PPHN} / \mathrm{PAH}$-disease related genes among 74 late preterm and term PPHN cases and 115 controls with matched clinical characteristics to further investigate the genetic etiology of PPHN. We identified 3 SNPs in CPS1 and 1 SNP in NOTCH3 associated with PPHN. The CPS1 SNPs rs192759073 and rs2229589 are synonymous variants, and rs1047883 is a missense variant. The heterozygous rs192759073 T allele was identified in 3 female PPHN patients and none of the controls. For rs1047883 and rs2229589, homozygous SNPs were found in 19 PPHN cases and 20 controls and heterozygous SNPs were found in 41 PPHN cases and 58 controls. The synonymous SNP rs1044008 in NOTCH3 was detected in three PPHN patients (heterozygous). These SNPs are reported to be associated with PPHN for the first time in this study. CPS1 (carbamoyl phosphate synthase 1) encodes one of the key enzymes located in mitochondria involved in the urea cycle. A functional deficiency in the CPS1 enzyme can affect the catalysis of the first step of the urea cycle and the generation of nitric oxide, which plays a critical role in regulating pulmonary vascular resistance [35]. Sixteen polymorphisms, including three in coding regions (rs1047891, rs2287599 and rs41272667) of CPS1 [3, 36], have been reported to be associated with PPHN. However, rs1047891 and rs2287599 were not significant in our cohort, and rs41272667 (close to the noncoding region) was not included in our study. The reason for this difference may be that the genetic risk factors for PPHN differ in different ethnic populations. NOTCH3 belongs to the Notch signaling pathway, which plays an important role in the regulation of cellular proliferation, differentiation and apoptosis. Heterozygous variants in $\mathrm{NOTCH} 3$ might affect cell proliferation and NOTCH3-HES5 signaling resulting in PAH [37]. Gene-level analysis also identified CPS1 and SMAD9 as genetic risk factors for PPHN.

There are several limitations to our study. We used clinical exome sequencing (with 16 PPHN/PAH diseaserelated genes included in the panel) for genetic testing, and the other 9 genes need to be further studied. Genetic risk polymorphisms are usually identified in noncoding regions, which cannot be detected using exome sequencing panels. However, exome sequencing provides more information for variants spread throughout genes than candidate SNP genotyping has provided in previous studies. Additionally, we could not study the association between nitric oxide metabolites and PPHN since the plasma concentrations of nitric oxide metabolites were not measured/recorded for all patients. 


\section{Conclusions}

In summary, this study identified 9 rare variants in PAH-related genes (TBX4, BMPR2, SMAD9, TGFB1, KCNA5 and TRPC6) in 9 PPHN patients in a Chinese cohort. We also revealed an association between the CPS1, NOTCH3 and SMAD9 genes and late preterm and term PPHN infants. Our findings have important clinical implications for the earlier detection of PPHN in preterm infants and provide more insight into the pathogenesis of PPHN and potential strategies for disease treatment.

\section{Additional files}

Additional file 1: Table S1 Reported PAH/PPHN related genes. (DOCX $18 \mathrm{~kb}$ )

Additional file 2: Table S2 Clinical phenotypes of 9 genetic positive PPHN patients. (DOCX $17 \mathrm{~kb}$ )

Additional file 3: Table S3 Gene-level analysis for rare variants. (DOCX $15 \mathrm{~kb}$ )

\section{Abbreviations}

ACMG: American College of Medical Genetics and Genomics;

ECMO: Extracorporeal membrane oxygenation; ExAC: Exome Aggregation Consortium; GnomAD: Genome Aggregation Database; HGMD: Human Gene Mutation Database; iNO: Inhaled nitric oxide; MAF: Minor allele frequency; MAS: Meconium aspiration syndrome; MOI: Maximal oxygenation index; NICU: Neonatal intensive care unit; OMIM: Online Mendelian Inheritance in Man; P/LP variants: Pathogenic or likely pathogenic variants; PAH: Pulmonary arterial hypertension; PAP: Pulmonary artery pressure; PPHN: Persistent pulmonary hypertension of the newborn; PVR: Pulmonary vascular resistance; RDS: Respiratory distress syndrome; SNP: Single nucleotide polymorphism; VUSs: Variants with unknown significance

\section{Acknowledgments}

The authors thank all the patients and their families for their participation in this study.

\section{Authors' contributions}

WZ and LY conceptualized and designed the study, supervised data collection and revised the manuscript. XL and MM analyzed and interpreted the data, drafted the manuscript, reviewed and revised the manuscript. $Y L$ and XD carried out the bioinformatic analysis. XC, LH, XH, YC and GC collected, supervised and reviewed the clinical data. All authors read and approved the final manuscript.

\section{Funding}

This study was supported by grants from China Postdoctoral Science Foundation (2017 M620133) and National Natural Science Foundation of China (81701498)

\section{Availability of data and materials}

The datasets used and/or analyzed during the current study are available from the corresponding author upon reasonable request.

\section{Ethics approval and consent to participate}

All guardians of the subjects included in this study were provided with appropriate informed consent. This study was approved by the ethics committee of the Children's Hospital of Fudan University and adhered to the Declaration of Helsinki.

\section{Consent for publication}

Not applicable.

\section{Competing interests}

The authors declare that they have no competing interests.

\section{Author details}

${ }^{1}$ Clinical Genetic Center, Children's Hospital of Fudan University, 399 Wanyuan Road, Shanghai 201102, China. 'Department of Neonatology, Children's Hospital of Fudan University, 399 Wanyuan Road, Shanghai 201102, China. ${ }^{3}$ Department of Pulmonology, Children's Hospital of Fudan University, 399 Wanyuan Road, Shanghai 201102, China. ${ }^{4}$ Key Laboratory of Birth Defects, Children's Hospital of Fudan University, 399 Wanyuan Road, Shanghai 201102, China.

Received: 31 January 2019 Accepted: 29 July 2019

Published online: 05 August 2019

References

1. Walsh-Sukys MC, Tyson JE, Wright LL, Bauer CR, Korones SB, Stevenson DK, Verter J, Stoll BJ, Lemons JA, Papile LA, et al. Persistent pulmonary hypertension of the newborn in the era before nitric oxide: practice variation and outcomes. Pediatrics. 2000;105:14-20.

2. Lai MY, Chu SM, Lakshminrusimha S, Lin HC. Beyond the inhaled nitric oxide in persistent pulmonary hypertension of the newborn. Pediatr Neonatol. 2018;59(1):15-23. https://doi.org/10.1016/j.pedneo.2016.09.011.

3. Pearson DL, Dawling S, Walsh WF, Haines JL, Christman BW, Bazyk A, Scott N, Summar ML. Neonatal pulmonary hypertension--urea-cycle intermediates, nitric oxide production, and carbamoyl-phosphate synthetase function. N Engl J Med. 2001;344:1832-8.

4. Kunig AM, Parker TA, Nogee LM, Abman SH, Kinsella JP. ABCA3 deficiency presenting as persistent pulmonary hypertension of the newborn. J Pediatr. 2007:151:322-4.

5. Byers HM, Dagle JM, Klein JM, Ryckman KK, McDonald EL, Murray JC, Borowski KS. Variations in CRHR1 are associated with persistent pulmonary hypertension of the newborn. Pediatr Res. 2012;71:162-7.

6. Mei M, Cheng G, Sun B, Yang L, Wang H, Sun J, Zhou W. EDN1 gene variant is associated with neonatal persistent pulmonary hypertension. Sci Rep. 2016;6:29877.

7. Abman SH, Hansmann G, Archer SL, Ivy DD, Adatia I, Chung WK, Hanna BD, Rosenzweig EB, Raj JU, Cornfield D, et al. Pediatric pulmonary hypertension: guidelines from the American Heart Association and American Thoracic Society. Circulation. 2015;132:2037-99.

8. Aldred MA, Vijayakrishnan J, James V, Soubrier F, Gomez-Sanchez MA, Martensson G, Galie N, Manes A, Corris P, Simonneau G, et al. BMPR2 gene rearrangements account for a significant proportion of mutations in familial and idiopathic pulmonary arterial hypertension. Hum Mutat. 2006;27:212-3.

9. Shintani M, Yagi H, Nakayama T, Saji T, Matsuoka R. A new nonsense mutation of SMAD8 associated with pulmonary arterial hypertension. J Med Genet. 2009;46:331-7.

10. Austin ED, Ma L, LeDuc C, Berman Rosenzweig E, Borczuk A, Phillips JA 3rd, Palomero T, Sumazin P, Kim HR, Talati MH, et al. Whole exome sequencing to identify a novel gene (caveolin-1) associated with human pulmonary arterial hypertension. Circ Cardiovasc Genet. 2012;5:336-43.

11. Ma L, Roman-Campos D, Austin ED, Eyries M, Sampson KS, Soubrier F, Germain M, Tregouet DA, Borczuk A, Rosenzweig EB, et al. A novel channelopathy in pulmonary arterial hypertension. N Engl J Med. 2013;369:351-61.

12. Harrison RE, Flanagan JA, Sankelo M, Abdalla SA, Rowell J, Machado RD, Elliott CG, Robbins IM, Olschewski H, McLaughlin V, et al. Molecular and functional analysis identifies ALK-1 as the predominant cause of pulmonary hypertension related to hereditary haemorrhagic telangiectasia. J Med Genet. 2003:40:865-71.

13. Chaouat A, Coulet F, Favre C, Simonneau G, Weitzenblum E, Soubrier F, Humbert M. Endoglin germline mutation in a patient with hereditary haemorrhagic telangiectasia and dexfenfluramine associated pulmonary arterial hypertension. Thorax. 2004;59:446-8.

14. Alano MA, Ngougmna E, Ostrea EM Jr, Konduri GG. Analysis of nonsteroidal antiinflammatory drugs in meconium and its relation to persistent pulmonary hypertension of the newborn. Pediatrics. 2001;107:519-23.

15. Wang K, Li M, Hakonarson H. ANNOVAR: functional annotation of genetic variants from high-throughput sequencing data. Nucleic Acids Res. 2010;38:e164.

16. McLaren W, Pritchard B, Rios D, Chen Y, Flicek P, Cunningham F. Deriving the consequences of genomic variants with the Ensembl API and SNP effect predictor. Bioinformatics. 2010;26:2069-70. 
17. Kumar P, Henikoff S, Ng PC. Predicting the effects of coding nonsynonymous variants on protein function using the SIFT algorithm. Nat Protoc. 2009;4:1073-81.

18. Adzhubei IA, Schmidt S, Peshkin L, Ramensky VE, Gerasimova A, Bork P, Kondrashov AS, Sunyaev SR. A method and server for predicting damaging missense mutations. Nat Methods. 2010;7:248-9.

19. Schwarz JM, Rodelsperger C, Schuelke M, Seelow D. MutationTaster evaluates disease-causing potential of sequence alterations. Nat Methods. 2010;7:575-6.

20. Richards S, Aziz N, Bale S, Bick D, Das S, Gastier-Foster J, Grody WW, Hegde

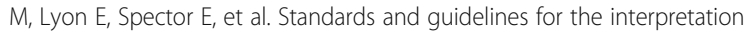
of sequence variants: a joint consensus recommendation of the American College of Medical Genetics and Genomics and the Association for Molecular Pathology. Genet Med. 2015;17:405-24.

21. Wu MC, Lee S, Cai T, Li Y, Boehnke M, Lin X. Rare-variant association testing for sequencing data with the sequence kernel association test. Am J Hum Genet. 2011;89:82-93.

22. Machado RD, Pauciulo MW, Thomson JR, Lane KB, Morgan NV, Wheeler L, Phillips JA 3rd, Newman J, Williams D, Galie N, et al. BMPR2 haploinsufficiency as the inherited molecular mechanism for primary pulmonary hypertension. Am J Hum Genet. 2001;68:92-102.

23. Navas P, Tenorio J, Quezada CA, Barrios E, Gordo G, Arias P, Lopez Meseguer M, Santos-Lozano A, Palomino Doza J, Lapunzina P, Escribano Subias P. Molecular analysis of BMPR2, TBX4, and KCNK3 and genotype-phenotype correlations in Spanish patients and families with idiopathic and hereditary pulmonary arterial hypertension. Rev Esp Cardiol (Engl Ed). 2016;69:1011-9.

24. Gentry LE, Nash BW. The pro domain of pre-pro-transforming growth factor beta 1 when independently expressed is a functional binding protein for the mature growth factor. Biochemistry. 1990;29:6851-7.

25. Fuloria M, Aschner JL. Persistent pulmonary hypertension of the newborn. Semin Fetal Neonatal Med. 2017;22:220-6

26. Fantozzi I, Platoshyn O, Wong AH, Zhang S, Remillard CV, Furtado MR, Petrauskene OV, Yuan JX. Bone morphogenetic protein-2 upregulates expression and function of voltage-gated $\mathrm{K}+$ channels in human pulmonary artery smooth muscle cells. Am J Physiol Lung Cell Mol Physiol. 2006;291: L993-1004.

27. Zhu N, Gonzaga-Jauregui C, Welch CL, Ma L, Qi H, King AK, Krishnan U, Rosenzweig EB, Ivy DD, Austin ED, et al. Exome sequencing in children with pulmonary arterial hypertension demonstrates differences compared with adults. Circ Genom Precis Med. 2018;11:e001887.

28. Levy M, Eyries M, Szezepanski I, Ladouceur M, Nadaud S, Bonnet D, Soubrier F. Genetic analyses in a cohort of children with pulmonary hypertension. Eur Respir J. 2016;48:1118-26.

29. Arora R, Metzger RJ, Papaioannou VE. Multiple roles and interactions of Tbx4 and Tbx5 in development of the respiratory system. PLoS Genet. 2012;8: e1002866

30. Jachec W, Foremny A, Domal-Kwiatkowska D, Smolik S, Tomasik A, Mazurek U, Wodniecki J. Expression of TGF-beta1 and its receptor genes (TbetaR I, TbetaR II, and TbetaR III-betaglycan) in peripheral blood leucocytes in patients with idiopathic pulmonary arterial hypertension and Eisenmenger's syndrome. Int J Mol Med. 2008;21:99-107.

31. Tsukamoto S, Mizuta T, Fujimoto M, Ohte S, Osawa K, Miyamoto A, Yoneyama K, Murata E, Machiya A, Jimi E, et al. Smad9 is a new type of transcriptional regulator in bone morphogenetic protein signaling. Sci Rep. 2014:4:7596.

32. Remillard CV, Tigno DD, Platoshyn O, Burg ED, Brevnova EE, Conger D, Nicholson A, Rana BK, Channick RN, Rubin LJ, et al. Function of Kv1.5 channels and genetic variations of KCNA5 in patients with idiopathic pulmonary arterial hypertension. Am J Physiol Cell Physiol. 2007;292: C1837-53.

33. Dietrich A, Chubanov V, Kalwa H, Rost BR, Gudermann T. Cation channels of the transient receptor potential superfamily: their role in physiological and pathophysiological processes of smooth muscle cells. Pharmacol Ther. 2006; 112:744-60.

34. Yu Y, Keller SH, Remillard CV, Safrina O, Nicholson A, Zhang SL, Jiang W, Vangala N, Landsberg JW, Wang JY, et al. A functional single-nucleotide polymorphism in the TRPC6 gene promoter associated with idiopathic pulmonary arterial hypertension. Circulation. 2009:119:2313-22.

35. Fineman JR, Wong J, Morin FC 3rd, Wild LM, Soifer SJ. Chronic nitric oxide inhibition in utero produces persistent pulmonary hypertension in newborn lambs. J Clin Invest. 1994;93:2675-83.
36. Kaluarachchi DC, Smith CJ, Klein JM, Murray JC, Dagle JM, Ryckman KK. Polymorphisms in urea cycle enzyme genes are associated with persistent pulmonary hypertension of the newborn. Pediatr Res. 2018;83:142-7.

37. Chida A, Shintani M, Matsushita $Y$, Sato H, Eitoku T, Nakayama T, Furutani $Y$, Hayama E, Kawamura Y, Inai K, et al. Mutations of NOTCH3 in childhood pulmonary arterial hypertension. Mol Genet Genomic Med. 2014;2:229-39.

\section{Publisher's Note}

Springer Nature remains neutral with regard to jurisdictional claims in published maps and institutional affiliations.
Ready to submit your research? Choose BMC and benefit from:

- fast, convenient online submission

- thorough peer review by experienced researchers in your field

- rapid publication on acceptance

- support for research data, including large and complex data types

- gold Open Access which fosters wider collaboration and increased citations

- maximum visibility for your research: over $100 \mathrm{M}$ website views per year

At BMC, research is always in progress.

Learn more biomedcentral.com/submissions 